\title{
Pre-operative fasting for intravenous conscious sedation used in dental treatment: are conclusions based on relative risk management or evidence?
}

IN BRIEF

- There is an historical association between sedation and general anaesthesia.

- Different centres impose varying protocols on pre-operative fasting for dental sedation.

- Guidelines are based on expert opinion and not randomised, controlled clinical trials.

- Current guidelines can be confusing.

- There is a need for high quality research to inform the debate on pre-operative fasting for dental sedation.

\author{
G. McKenna ${ }^{1}$ and S. Manton ${ }^{2}$
}

\begin{abstract}
Intravenous sedation is a widely used pharmacological method of patient management commonly used in dental surgery for the treatment of anxious patients. Variety exists in fasting regimes between different centres offering dental sedation, with some advocating starvation in line with general anaesthesia protocols and others not enforcing starvation at all. The currently available guidelines on fasting protocols are ambiguous and open to interpretation partly because they are based on expert opinion rather than evidence-based research. This article reviews the available evidence on the subject of pre-operative fasting and discusses current guidelines.
\end{abstract}

\section{Introduction}

Sedative techniques have been used successfully to facilitate the practice of dentistry for many years. In the dental setting, these methods are viewed as adjuncts to patient management, rather than techniques which negate the need for co-operation from the patient or reduce the requirement for communication between the dental team and the patient. The definition of 'conscious sedation' quoted below which binds the dental profession in the United Kingdom is that accepted by the General Dental Council, the Standing Dental Advisory Committee, the National Dental Advisory Committee and the Dental Sedation Teachers Group:

A technique in which the use of a drug, or drugs, produces a state of depression of the central nervous system enabling treatment to be carried out, but during which communication is maintained and the modification of the patient's state of mind is such that the patient will respond to command throughout

\footnotetext{
*Senior House Officer, ${ }^{2}$ Consultant and Honorary Senior Lecturer in Restorative Dentistry, Dundee Dental Hospital, University of Dundee, Dundee, DD1 4HN *Correspondence to: Dr Gerald McKenna Email: g.mckenna@ucc.ie
}

\section{Refereed Paper}

Accepted 27 May 2008

DOI: $10.1038 /$ sj.bdj.2008.692

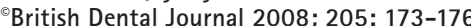

the period of sedation. Techniques used should carry a margin of safety wide enough to render unintended loss of consciousness unlikely. ${ }^{1-6}$

Intravenous sedation is the most commonly used method of sedation for the provision of dental care. By administering drugs directly into the circulation the onset of sedation is rapid, the dose can be titrated according to patient response, after initial venepuncture administration is painless, intravenous access is preserved and recovery time is short. The most commonly used drugs in the practice of intravenous sedation are the benzodiazepines. Midazolam is a water-soluble imidazodiazepine with an elimination half life of 90-150 minutes which has been marketed in the United Kingdom since 1984. Propofol is a synthetic sedative hypnotic used extensively in the induction of general anaesthesia. However, it is also a very effective dental sedative agent which can be administered under the control of an operator or the patient. At this time propofol is not licensed for administration outside the hospital environment, and its use is restricted to those who have the necessary training and experience to deal with the unconscious patient. In addition, a number of experienced and appropriately trained sedation practitioners in the UK use other advanced sedation techniques employing combinations of intravenous drugs, including opioids.

\section{Applications of conscious sedation}

Sedation is considered beneficial for a wide variety of patients. The greatest benefit is for the anxious patient who cannot tolerate routine dental treatment under local anaesthesia alone, usually as a result of anxiety regarding the treatment. In The Adult Dental Health Survey of 1998 it was reported that 33\% of those interviewed rated fear as the major barrier to dental care. ${ }^{7}$ For these patients, treatment under sedation may be required for various aspects of dental care ranging from a clinical examination to complex interventions. Other psychological problems which are amenable to sedation include uncontrollable retching or gagging and persistent fainting after administration of local anaesthesia. The majority of patients will accept routine dental care under local anaesthesia, but may request some additional help in coping with specific items of dental treatment. Most commonly this relates to oral surgical procedures such as removal of impacted third molar teeth, ${ }^{8}$ but sedation is increasing in popularity for the surgical placement of dental implants.

There are a number of medical conditions where the patient, despite 
wishing to cooperate with dental care, finds it impossible to do so. Such conditions can range from involuntary twitches of specific muscle groups to generalised movements of all the muscles of the body, as can be found in Parkinson's disease. Sedation is of great value for these patients as it decreases anxiety and can provide a muscle relaxant action, reducing or occasionally eliminating the involuntary movements. Patients with learning difficulties can often have problems co-operating with treatment despite a perceived willingness to do so. If the patient is capable of understanding the treatment on offer and sedation will help, then the chances of success can be good. ${ }^{9}$

The acute episodes of medical conditions, such as ischaemic heart disease, asthma and epilepsy (which present intermittent or episodic symptoms) can be precipitated, or aggravated by stress. A history of one of these conditions accompanied by anxiety regarding dental treatment can increase the probability of a patient suffering an acute episode of their medical condition in the dental surgery. Sedation should be considered for patients at risk of medical events during dental or medical treatment. $^{10}$

\section{Relationship with general anaesthesia}

Modern sedation has undoubtedly reduced the number of patients who require general anaesthesia to tolerate dental treatment. Historically however, sedation and general anaesthesia are closely linked and are both considered within the wide spectrum of pharmacological patient management. In many centres anaesthetic colleagues have an input into conscious sedation including its administration. As a consequence of this close relationship, patients undergoing conscious sedation have fasted historically in accordance with general anaesthesia protocols. Fasting before general anaesthesia aims to reduce the volume and acidity of stomach contents during surgery, thus reducing the risk of regurgitation and aspiration syndrome. The latter causes cyanosis, respiratory difficulties including airway obstruction and possibly death depending on the severity of pulmonary damage. ${ }^{11}$ The common use of fasting regimes may be explained by a number of factors. A single protocol adopted by a department can help to avoid confusion and mistakes where sedation and general anaesthesia are both offered. Therefore, protocols have often been slow to change. In addition, some would argue that nervous patients undergoing dental sedation may be considered more likely to vomit during the procedure or in recovery and thus an empty stomach could prevent further complications.

\section{Current guidelines on preoperative fasting}

There is currently wide disparity in the pre-operative fasting times for patients undergoing intravenous conscious sedation throughout the United Kingdom. A contributing factor in this situation has been the variation in guidelines published. The Poswillo Report (1990) advised a pre-operative starvation period of four hours for fluids and solids prior to general anaesthesia and sedation. ${ }^{12}$ In 2000, the Society for the Advancement of Anaesthesia in Dentistry commissioned an independent report on standards for conscious sedation in dentistry. ${ }^{2}$ This report advised patients to consume light food prior to sedation and concluded that starvation was undesirable. The Standing Dental Advisory Committee published guidelines in $2003^{3}$ and these stated that fasting is not normally required but a patient should be advised to take only light food and clear nonalcoholic fluids before a conscious sedation appointment. However, the same document also stated that some authorities may recommend the same fasting requirements as for those undergoing a general anaesthetic in view of the potential depression of upper airway reflexes.

The most recent publication entitled Conscious sedation in dentistry produced by the Scottish Dental Clinical Effectiveness Programme in 2006 stated that there was insufficient evidence to inform the debate about whether fasting prior to treatment contributed to patient safety for conscious sedation in dentistry. However, the report went on to say that based on a consensus of expert opinion, fasting for conscious sedation is not required unless there is a specific indication. It was recommended that patients should be advised to eat normally on the day of their appointment, but should avoid alcoholic drinks and large meals. ${ }^{13}$

\section{What is the evidence in support of pre-operative fasting?}

The importance of fasting is to prevent a rare but serious complication known as aspiration pneumonitis which was initially reported in obstetric patients. In 1946, Mendelson described two different aspiration syndromes following analysis of patients who had aspirated during obstetric anaesthesia. He reported 66 patients who had aspirated under anaesthesia over a 13 year period and described that aspiration of solid material caused an obstructive reaction which potentially resulted in asphyxia and death, whereas aspiration of gastric fluids caused an asthmatic type reaction resulting in bronchoconstriction and inflammation in the lower airways. ${ }^{11}$

After a series of animal experiments, Mendelson concluded that regurgitated gastric acid was responsible for the pulmonary damage observed with aspiration syndrome and as a result he introduced the concept of fasting prior to anaesthesia. ${ }^{11}$ However, later studies reported that patients undergoing general anaesthesia whilst pregnant were statistically more likely to suffer from aspiration pneumonitis due to delayed gastric emptying. ${ }^{14}$ Olssen et al. observed that patients most often affected were those at the extremes of age and the highest mortality was associated with those who were in poor physical condition. ${ }^{15}$ The paper identified several factors indicating an increased risk for aspiration, including upper abdominal and emergency abdominal surgery. Patient risk factors included obesity, diabetes, peptic ulcer disease, incompetent lower oesophageal sphincter, narcotic medication, increased risk of delayed gastric emptying and regurgitation. In contrast, however, other authors have shown that obese patients without oesophageal or gastric pathology had less gastric juice and less acid than in leaner patients. ${ }^{16}$

Researchers have attempted to determine a minimum volume and $\mathrm{pH}$ of 
gastric contents required to produce aspiration pneumonitis but without a definitive answer. It has been reported that in some patients even a minute volume of gastric contents can lead to aspiration. ${ }^{17}$ Coupled with this is the identification of airway manipulation as one of the most frequent causes of aspiration syndrome. During the placement and removal of an endotracheal tube, air can be blown into the stomach causing coughing and gatro-oesophageal reflux which in turn can precipitate aspiration. ${ }^{18}$ Whilst airway management is not normally part of conscious sedation, it could form part of the response to an emergency situation. The principal side effect seen with the acute administration of large doses of benzodiazepines is that of respiratory depression. A study by Murphy et al. carried out in 1994 showed a depression of airway reflex sensitivity with diazepam and midazolam. ${ }^{19}$ A number of volunteers were administered a large bolus dose of the sedative agent and respiratory effects were observed. In addition, the respiratory depression produced by benzodiazepines had a synergistic relationship with respiratory depression produced by other drugs particularly the opioids. However, the evidence from this study is not universally accepted due to the small patient sample and the large doses of drugs administered.

\section{What is the evidence opposing pre-operative fasting?}

Aspiration pneumonitis, although a potentially lethal complication, is rare. Mendelson observed 44,016 patients and reported that just $0.15 \%$ suffered from the complication whilst under general anaesthesia. ${ }^{11}$ Olsson et al. carried out a retrospective study of more than 185,000 anaesthetic outcomes. The overall incidence of aspiration syndrome was reported as 1 in 2,131 patients with mortality after aspiration at 5\%. ${ }^{15}$

There is very little evidence within published medical literature which concludes that pre-operative fasting results in a decreased incidence of adverse effects in patients undergoing conscious sedation. The risk of aspiration during conscious sedation has not been quantified and there are no reports of such cases in the literature. In a study of chloral hydrate sedation used on 200 infants, fasting was associated with an increased dose of drug required to produce the desired clinical effects. The authors concluded that this was due to the fact that the infants were more irritable and thus more difficult to sedate after fasting. ${ }^{20}$ Pena et al. carried out a prospective analysis of adverse effects of conscious sedation within a paediatric emergency department. Of 1,180 patients, 27 had an adverse event (2.3\%). Of the 27 patients, five vomited but there was no clinical evidence of aspiration reported in any case. ${ }^{21}$ The patients had been sedated using a combination of intravenous midazolam and fentanyl; a mixture not routinely used in the UK for dental sedation.

Recent studies have indicated that fasting during the pre-operative period can induce a metabolic state that is unfavourable for patients. Glycogen stores are depleted after an overnight fast and therefore patients use muscle for gluconeogenesis. ${ }^{22}$ This results in reduced muscle strength after surgery. ${ }^{23}$ Overnight fasting has also been shown to induce postoperative insulin resistance. ${ }^{24}$ It is thought that carbohydrate availability and the metabolic setting of the fed state are important factors in postoperative recovery. ${ }^{25}$ Pre-operative carbohydrate treatment has been shown to produce the change in metabolism that usually occurs when breakfast is eaten. This causes an insulin release which turns off the overnight fasting metabolic state. The development of carbohydrate drinks with low osmolality ${ }^{24}$ enable them to pass through the stomach in only 90 minutes. ${ }^{26}$ Human studies have shown that provision of such a carbohydrate drink prior to major elective abdominal surgery may attenuate the reduction of muscle mass after surgery. ${ }^{27}$ There have also been varying claims that a pre-operative carbohydrate drink can reduce pre-operative thirst, hunger, anxiety and improve overall well being. ${ }^{25}$

Guidelines for fasting prior to general anaesthesia have been relaxed in a number of countries in recent years. Norway $^{28}$ first adopted new and more liberal guidelines in 1993, followed by the United Kingdom, ${ }^{29}$ the United States of America, ${ }^{30}$ Sweden and Denmark. ${ }^{24}$ Such guidelines now recommend that for adults undergoing elective procedures, the pre-operative fasting period is two hours for clear fluids and six hours for solid foods. Allowing patients to have these fluids has reduced pre-operative thirst, headaches, irritation, discomfort and postoperative nausea and vomiting. ${ }^{21,31}$ In addition, patients given water pre-operatively were found to have a significantly lower volume of gastric contents than those who followed overnight fasting regimes, as the stimulation from the drink increased the rate of gastric emptying. Since the implementation of these guidelines there has not been a significant increase in the incidence of pulmonary aspiration or other unwanted side effects. In fact, there is no indication that fluids permitted up to 90 minutes pre-operatively lead to an increased risk of regurgitation and aspiration. ${ }^{27}$

It is commonly thought that stress or anxiety increases gastric acid secretion and may therefore delay gastric emptying. This could be very relevant to patients attending for conscious sedation, especially those anxious about dental treatment. However, studies have failed to illustrate a correlation between anxiety state and gastric emptying. Haavik et al. investigated the relationship but determined that gastric volume and $\mathrm{pH}$ did not correlate with anxiety levels. ${ }^{32}$

\section{Conclusion}

Conscious sedation is a very useful and necessary adjunct for the provision of dental treatment for a wide variety of patients. Sedation and general anaesthesia are historically linked but important differences exist. One area where common ground has traditionally persisted is that of pre-operative fasting. Starving prior to general anaesthesia aims to reduce the possibility of regurgitation and aspiration pneumonitis by minimising gastric volume and $\mathrm{pH}$. However, after reviewing the medical literature available there is little strong evidence to support fasting prior to intravenous conscious sedation. The reported risk of aspiration pneumonitis under general anaesthesia is very low and the relative risk under conscious sedation 
is substantially less especially where protective reflexes are maintained, airway manipulation is not used and the technique is only utilised for ASA Classification I and II patients. In addition, evidence suggests that after relaxation of fasting protocols prior to general anaesthesia, the incidence of aspiration has not significantly increased. In fact the literature illustrates that consumption of carbohydrate prior to anaesthesia may well improve patient well being and even expedite recovery. Guidelines available generally acknowledge that there is little need for starvation prior to treatment under conscious sedation. However, some of the published advice is ambiguous and open to interpretation. This is at least partly responsible for the variation in fasting regimes between different units throughout the United Kingdom. Some district general hospitals with anaesthetic input will impose extended fasting regimes prior to conscious sedation in line with general anaesthesia recommendations. Such protocols appear to be unnecessary, may be unpleasant for the patient and may adversely affect recovery after the procedure.

Variations in protocols illustrate that the debate on pre-operative fasting prior to conscious sedation has not yet fully been resolved. Standardised regimes and regulations should be established on the basis of available scientific evidence linked to nationally recognised guidelines. Therefore, in order to solve this debate, there is a need for high quality randomised controlled trials to be carried out within an appropriate governance framework, thus facilitating evidence-based practice.

1. A Conscious Decision: A Review of the Use of General Anaesthesia and Conscious Sedation in Primary Dental Care. Report of a Group Chaired by the Chief Medical and Chief Dental Officer. Department of Health, 2000.

2. Standards in Conscious Sedation for Dentistry. Report of an Independent Expert Working Group. Society for the Advancement of Anaesthesia in Dentistry, 2000.

3. Conscious Sedation in the Provision of Dental Care. Report of an Expert Group on Sedation for Dentistry. Standing Dental Advisory Committee, Department of Health, 2003.

4. Emergency Dental Drugs - National Dental Advisory Committee. Scottish Office Department of Health, 1999.

5. Standards for Dental Professionals. General Dental Council, 2005

6. Training in Conscious Sedation for Dentistry. Dental Sedation Teachers Groups, 2005.

7. Nuttall N M, Bradnock G, White D, Morris J, Nunn J. Dental attendance in 1998 and implications for the future. Br Dent J 2001; 190: 177-182.

8. Luyk N H, Whitely B D. Efficacy of oral midazolam prior to intravenous sedation for the removal of third molars. Int J Oral Maxillofac Surg 1991; 20: 264-267.

9. Stephens A J, Sapsford D J, Curzon M E. Intravenous sedation for handicapped dental patients: a clinical trial of midazolam and propofol. Br Dent $J$ 1993; 175: 20-25

10. Meehan J G, Robb N D, Seymour R A. Pain and anxiety control for the conscious dental patient. Oxford University Press, 1998.

11. Mendelson C L. The aspiration of stomach contents into the lungs during obstetric anaesthesia. Am J Obstet Gynecol 1946; 52: 191-205.

12. Poswillo D E. General anaesthesia, sedation and resuscitation in dentistry. The Report of an Expert Working Party. London: Department of Health, 1990.

13. Conscious Sedation in Dentistry, Dental Clinical Guidance. Scottish Dental Clinical Effectiveness Programme, May 2006.

14. Roberts R B, Shirley M A. Reducing the risk of acid aspiration during caesarean section. Anesth Analg 1974; 53: 859-868.

15. Olsson G L, Hallen B, Hambraeus Jonzon K. Aspiration during anaesthesia: a computer-aided study of 185,358 anaesthetics. Acta Anaesthesio/ Scand 1986; 30: 84-92.

16. Harter R L, Kelly W B, Kramer M G, Perez C E, Dzwonczyk R R. A comparison of the volume and $\mathrm{pH}$ of gastric contents of obese and lean surgical patients. Anesth Analg 1998; 86: 147-152.

17. Green S M, Krauss B. Pulmonary aspiration risk during emergency department procedural sedation - an examination of the role of fasting and sedation depth. Acad Emerg Med 2002; 9: 35-42.

18. Ljungqvist 0 , Soreide E. Preoperative fasting. BrJ Surg 2003; 90: 400-406.

19. Murphy P J, Erskine R, Langton J A. The effect of intravenously administered diazepam, midazolam and flumazenil on the sensitivity of upper airway reflexes. Anaesthesia 1994; 49: 105-110.

20. Keidan I, Gozal D, Minuskin T, Weinberg M et al. The effect of fasting practice on sedation with chloral hydrate. Pediatr Emerg Care 2004; 20: 805-807.

21. Pena B M G, Krauss B. Adverse events of procedural sedation and analgesia in a paediatric emergency department. Ann Emerg Med 1999; 34: 483-491.

22. Ljungqvist 0 . To fast or not to fast before surgical stress. Nutrition 2005; 21: 885-886.

23. Diks J, van Hoorn D E C, Nijveldt R J, Boelens P G et al. Preoperative fasting: an outdated concept? JPEN J Parenter Enteral Nutr 2005; 29: 298-304.

24. Ljungqvist 0 , Nygren J, Hausel J, Thorell A. Preoperative nutrition therapy - novel developments. Scand J Nutrition 2000; 44: 3-7.

25. Nygren J, Thorell A, Jacobsson H, Larsson S et al. Preoperative gastric emptying; effect of anxiety and oral carbohydrate administration. Ann Surg 1995: 222: 728-734.

26. Yuill K A, Richardson R A, Davidson H I M, Garden 0 J, Parks R W. The administration of an oral carbohydrate-containing fluid prior to major elective upper-gastrointestinal surgery preserves skeletal muscle mass postoperatively - a randomised clinical trial. Clin Nutr 2005; 24: 32-37.

27. Hausel J, Nygren J, Lagerkranser M, Hellstrom P $M$ et al. A carbohydrate-rich drink reduces preoperative discomfort in elective surgery patients. Anesth Analg 2001; 93: 1344-1350.

28. Soreide E, Raeder J C. Norwegian national consensus guidelines for preoperative fasting in elective surgery. Acta Anaesthesio/ Scand 1998; 42: 1188-1191.

29. Perioperative fasting in adults and children. The Royal College of Nursing. November 2005.

30. Practical guidelines for preoperative fasting and the use of pharmacological agents to reduce the risk of pulmonary aspiration: application to healthy patients undergoing elective procedures: a report by the American Society of Anaesthesiologists Task Force on Preoperative Fasting. Anesthesiology 1999; 90: 896-905.

31. Smith A F, Vallance H, Slater R M. Shorter preoperative fluid fasts reduce postoperative emesis. Br Med J 1997; 314: 1486.

32. Haavik P E, Soreide E, Hofstad B, Steen P A. Does preoperative anxiety influence gastric fluid volume and acidity? Anesth Analg 1992; 75: 91-94. 